Proceedings

\title{
Study on the Synthesis of Some (Un)Substituted 2- Amino-4-aryl-7-hydroxy-4H-chromene-3-carbonitriles in the Water Medium ${ }^{\dagger}$
}

\author{
Nguyen Dinh Thanh 1,*, Do Son Hai 1,2, Pham Thi Thu Hien 1, Vu Thi Ngoc Bich 1, \\ Vu Ngoc Toan ${ }^{1,3}$, Hoang Thi Kim Van ${ }^{1,4}$ and Tran Thi Thanh Van ${ }^{1}$ \\ 1 Faculty of Chemistry, VNU University of Science (Vietnam National University, Ha Noi), \\ 19 Le Thanh Tong, Hoan Kiem, Ha Noi 1906, Vietnam; dosonhai82@gmail.com (D.S.H.); \\ phamthithuhien_t58@hus.edu.vn (P.T.T.H.); bichxu1195@gmail.com (V.T.N.B.); \\ vntoanchem@gmail.com (V.N.T.); hoangvan8868@gmail.com (H.T.K.V.); tvche@yahoo.com (T.T.T.V.) \\ 2 Lab of Profession Chemistry, Institute of Biochemical Technology and Profession Documents, \\ General Department 4 (Ministry of Public Security), 80 Tran Quoc Hoan, Cau Giay, Ha Noi 11300, Vietnam \\ 3 Institute for Chemistry and Materials, Military Institute of Science and Technology, 17 Hoang Sam, \\ Cau Giay, Ha Noi 100000, Vietnam \\ 4 Viet Tri University of Industry, Tien Kien, Lam Thao, Phu Tho 290000, Vietnam \\ * Correspondence: nguyendinhthanh@hus.edu.vn; Tel.: +84-04-3826-1853; Fax: +84-04-3824-1140 \\ + Presented at the 22nd International Electronic Conference on Synthetic Organic Chemistry, \\ 15 November-15 December 2018; Available Online: https://sciforum.net/conference/ecsoc-22. \\ Published: 14 November 2018
}

\begin{abstract}
An efficient and simple synthesis of substituted 2-amino-4H-chromene-3-carbonitriles was developed by the one-pot and three-component reaction of a mixture of corresponding substituted benzaldehydes, resorcinol, and malononitrile in the presence of sodium carbonate solution as a catalyst at room temperature.
\end{abstract}

Keywords: $4 H$-chromene; one-pot reaction; multi-component reaction; propargyl ether; singlecrystal X-ray structure

\section{Introduction}

Recently, multi-component coupling reactions (MCRs) have received interest in organic synthesis due to their conveniences and benefits [1]. In these reactions, three or more starting materials react with each other to form a product, where all or most of the atoms contribute to the newly formed product. Essentially, in an MCR, a product is created by putting components or members together according to a cascade of elementary chemical reactions [1]. Histologically, the MCRs were discovered by Adolph Strecker in 1860 [2] with the synthesis of $\alpha$-amino acid, involving the reaction of potassium cyanide, ammonium chloride, and an aldehyde to make an alpha amino acid, and nowadays are continuously developed organically and synthetically in laboratories [3,4].

The organic reactions that are carried out in an aqueous medium and at room temperature are of great interest to synthetic chemists [5,6]. Water is an environmental, nonflammable, nonhazardous, inexpensive, and naturally available solvent. Water also gives the benefits of simple workup and purification by simple phase separation techniques. Therefore, organic reactions carried out in aqueous media are an attractive and interesting area of green chemistry $[7,8]$. Additionally, the performance of the organic reactions at room temperature helps mitigate the decomposition of the product (if available) or the formation of unwanted products [9]. 
$4 \mathrm{H}$-Chromene derivatives and compounds with chromene moiety have notable usage in organic synthesis due to their biological activities [10]. These compounds function as the important pharmacophores, which are associated with a broad range of pharmacological activities. They are antimicrobial [11], hypolipidemic [12], anti-inflammatory [13,14], anti-proliferative [15], antioxidant [11,16], anticoagulant [17], and anti-leishmanial [18], antitumor [15], anti-diabetic [19], cytotoxicity $[10,20]$, and anticancer $[16,20]$ agents.

It is known that 2-amino-4H-chromene compounds have been synthesized from (un)substituted benzaldehydes, malononitrile, and (un)substituted resorcinols [21,22]. Chromene ring is formed in the presence of some inorganic and organic catalysts, such as $\mathrm{K}_{2} \mathrm{CO}_{3}$ in water [23], $\mathrm{Ca}(\mathrm{OH})_{2}$ [24], nano $\mathrm{ZnO}$ [25], nanocrystalline $\mathrm{MgO}$ in aqueous media [26], silica gel supported polyamine [27], ammonium acetate [28], and potassium phthalimide [29]. Some ionic liquids are also used as catalysts for this purpose, for example, [2-Eim]OAc [30], basic ionic liquids from triethylenetetramine or ethylenediamine, and TFA (trifluoroacetic acid) [31]. Monodisperse Pd nanomaterials anchored graphene oxide [32] and bimetallic Pd-Ru/graphene oxide-based catalysts [33] are also applied in the one-pot three-component synthesis of 2-amino- $4 \mathrm{H}$-chromene derivatives. In some cases, the $4 \mathrm{H}$ chromene ring is closed in one-pot and solvent-free synthesis using grindstone chemistry [34] (by simple grinding). Bovine serum albumin is also used as a catalyst in a domino reaction for the synthesis of 2-amino-4H-chromene derivatives [35]. 2,2,2-trifluoroethanol is used, too, as a metal-free and reusable medium in a facile and efficient synthesis of 2-amino-3-cyano- $4 \mathrm{H}$-chromenes and tetrahydrobenzo[b]pyrans [36]. The ultrasound irradiation is applied under procedure without catalyst [37] or catalyzed by $\mathrm{Fe}_{3} \mathrm{O}_{4}$-functionalized nanoparticles with chitosan [38], by glycine in aqueous medium under sonic conditions [39], or by electrochemically-induced multi-component condensation in the presence of $\mathrm{NaBr}[40]$.

Therefore, in this article, we report on the synthesis of some (un)substituted 2-amino-7-hydroxy$4 H$-chromene-3-carbonitriles via a one-pot three-component reaction in aqueous media (Scheme 1).

\section{Results and discussion}

For the purpose of applying an environmental and cheap solvent, we used water as a solvent in the synthesis of compounds of (un)substituted 2-amino-7-hydroxy-4-aryl-4H-chromene-3carbonitriles (Scheme 1). The model reaction was made with unsubstituted benzaldehyde 1a (Table 1). The base, reaction time, and reaction temperature optimizations for yields are listed in Table 1. The different inorganic and organic basic catalysts were used in the catalyst surveys, such as $\mathrm{NaOH}$, $\mathrm{NaHCO}_{3}, \mathrm{Na}_{2} \mathrm{CO}_{3}, \mathrm{~K}_{2} \mathrm{CO}_{3}$, ammonium acetate, 4-dimethylaminopyridine (DMAP), triethylamine, and piperidine, and the reactions were examined in water at room temperature.

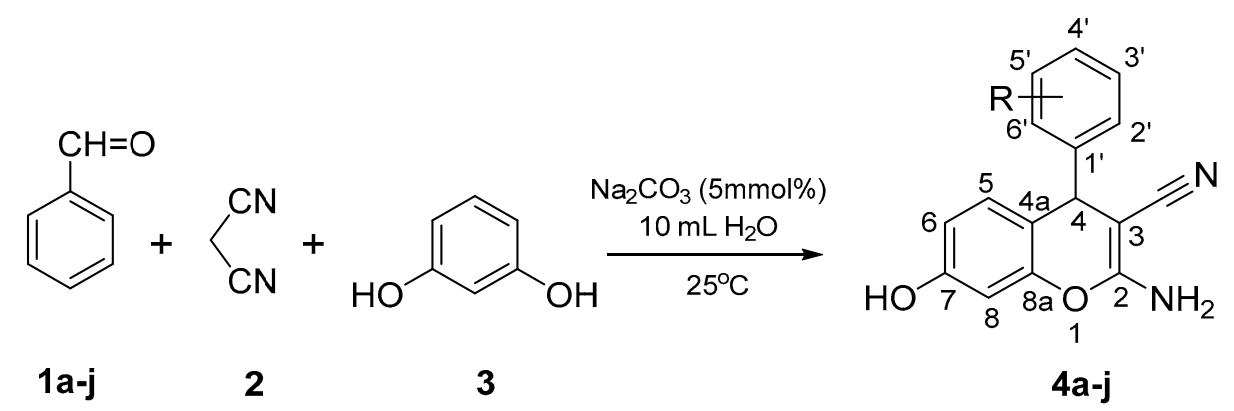

Scheme 1. Synthetic route for substituted 2-amino-7-hydroxy-4-aryl-4H-chromene-3-carbonitriles. Condition reactions: See Table 2.

In general, the inorganic bases gave the product $4 \mathbf{a}$ in higher yields (Entries 1-4), while the organic bases gave the product $\mathbf{4 a}$ in lower yields (Entries $5-8$ ). We found that sodium carbonate (with 0.1 equivalent amount, Entries 3) in $10 \mathrm{~mL}$ of water provided the target product $4 \mathrm{a}$ in $90 \%$ yield. Therefore, sodium carbonate was chosen for further investigations in water at different times and temperatures (Entries 8-15). 
Table 1. One-pot synthesis of $4 \mathrm{H}$-chromene in presence of various bases a ${ }^{\text {. }}$

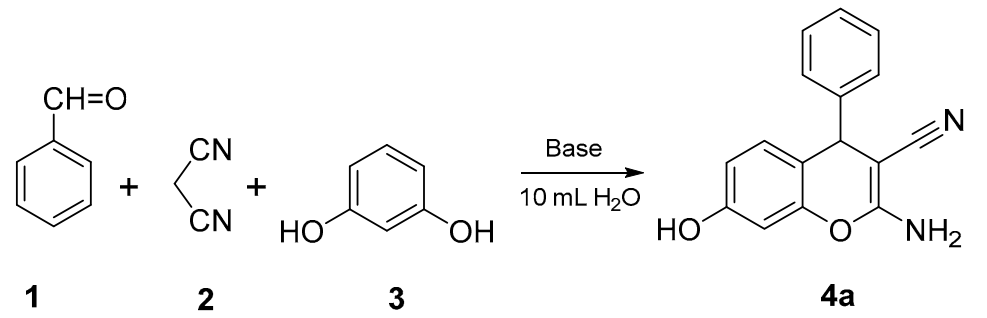

\begin{tabular}{ccccc}
\hline Entry & Bases (equiv) & Time (min) & Temp. $\left({ }^{\circ} \mathbf{C}\right)$ & Yield of $4 \mathbf{a}^{\mathbf{b}} \mathbf{( \% )}$ \\
\hline 1 & $\mathrm{NaOH}(0.1)$ & 2 & 25 & 58 \\
2 & $\mathrm{NaHCO}_{3}(0.1)$ & 2 & 25 & 65 \\
3 & $\mathrm{Na}_{2} \mathrm{CO}_{3}(0.1)$ & 2 & 25 & 90 \\
4 & $\mathrm{~K}_{2} \mathrm{CO}_{3}(0.1)$ & 2 & 25 & 76 \\
5 & Ammonium acetate $(0.1)$ & 2 & 25 & 67 \\
6 & $\mathrm{DMAP}(0.1)$ & 2 & 25 & 50 \\
7 & $\mathrm{Et} 3 \mathrm{~N}(0.1)$ & 2 & 25 & 45 \\
8 & $\mathrm{Piperidine}(0.1)_{2}$ & 2 & 25 & 55 \\
9 & $\mathrm{Na}_{2} \mathrm{CO}_{3}(0.05)$ & 2 & 25 & 90 \\
10 & $\mathrm{Na}_{2} \mathrm{CO}_{3}(0.15)$ & 2 & 25 & 88 \\
11 & $\mathrm{Na}_{2} \mathrm{CO}_{3}(0.2)$ & 2 & 25 & 87 \\
12 & $\mathrm{Na}_{2} \mathrm{CO}_{3}(0.1)$ & 2 & 70 & 80 \\
13 & $\mathrm{Na}_{2} \mathrm{CO}_{3}(0.1)$ & 2 & Reflux & 70 \\
14 & $\mathrm{Na}_{2} \mathrm{CO}_{3}(0.1)$ & 3 & 25 & 91 \\
15 & $\mathrm{Na}_{2} \mathrm{CO}_{3}(0.1)$ & 4 & 25 & 90 \\
\hline
\end{tabular}

a Reaction conditions: The reaction was performed by using $2 \mathrm{mmol}$ of $\mathbf{1}, 2 \mathrm{mmol}$ of 2 , and $2 \mathrm{mmol}$ of 3 , in the presence of $10 \mathrm{~mol} \%$ or $5 \mathrm{~mol} \%$ of basic additive in $10 \mathrm{~mL}$ of water under different temperature conditions. ${ }^{\mathrm{b}}$ Isolated yields.

When the concentration of this catalyst was half reduced, the yield of 4a did not change (in $90 \%$ yield in Entry 9). We found that increasing amounts of this catalyst, from 0.1 equiv. (Entry 3) to 0.15 equiv. (Entry 10) to 0.2 equiv. (Entry 11), resulting in the slight reduction of reaction yields $(88 \%$ in Entry 10 and $87 \%$ in the Entry 11). On the other hand, raising the temperature of the reaction from 25 ${ }^{\circ} \mathrm{C}$ (Entry 3) to $70{ }^{\circ} \mathrm{C}$ (Entry 12) in the reflux-heating method (Entry 13) lead to reduced product yields of $4 a$, to $80 \%$ and $70 \%$, respectively. The reaction time did not significantly change the yields of the products. For example, in Entries 14 and 15, the yields of 4a were $91 \%$ (for $180 \mathrm{~min}$ ) and $90 \%$ (for 240 $\mathrm{min})$, respectively. Hence, sodium carbonate in the amount of 0.05 equiv. in $10 \mathrm{~mL}$ of water was used in the synthesis of all the other chromenes $\mathbf{4 b} \mathbf{b} \mathbf{l}$ (Table 2).

The absorption band that appeared in regions at $v=3500-3450 \mathrm{~cm}^{-1}$ belonged to the hydroxyl group, and two other absorption bands that appeared in regions $3420-3250 \mathrm{~cm}^{-1}$ in IR spectra of $4 \mathrm{H}$ chromenes $4 \mathrm{a}-1$ confirmed the presence of the amino group in these molecules. The nitrile functional group had the absorption band at 2203-2188 $\mathrm{cm}^{-1}$. All ${ }^{1} \mathrm{H}$ NMR spectra of chromenes $4 \mathrm{a}-1 \mathrm{l}$ had characteristic signals at $\delta=4.95-4.50 \mathrm{ppm}$, with an integral height of one proton; this signal belongs to a proton on position 4 and could be used as the identification sign for $4 \mathrm{H}$-pyrans and $4 \mathrm{H}$ chromenes. In addition, the chemical shift appearing at $\delta=9.70-9.60 \mathrm{ppm}$ confirmed the presence of a hydroxyl group on position 7 of the chromene ring. The amino group on position 2 of chromenes 4a-1 had a resonance signal at $\delta=6.84-6.83 \mathrm{ppm}$. The carbon atom at position 2 had a chemical shift downfield at $\delta=161.4-160.1 \mathrm{ppm}$, due to the electron-withdrawing influence of an oxygen atom in the chromene ring. The carbon atom in the nitrile group showed a resonance signal at $\delta=121.2-119.6$ $\mathrm{ppm}$. This evidence proved that chromenes 4a-1 were formed through three component reactions between substituted benzaldehydes $\mathbf{1 a}-\mathbf{1}$, malononitrile $\mathbf{2}$, and resorcinol $\mathbf{3}$, in the presence of sodium carbonate as a catalyst. 
Table 2. Sodium carbonate catalyzed the one-pot synthesis of $4 H$-chromene derivatives (4a-1) a.

\begin{tabular}{cccccc}
\hline Entry & $\mathbf{R}$ & & Time (h) & Temp. $\left({ }^{\circ} \mathbf{C}\right)$ & Yield $^{\mathbf{b}} \mathbf{( \% )}$ \\
\hline 1 & $\mathrm{H}$ & $\mathbf{4 a}$ & 2 & 25 & 90 \\
2 & $4-\mathrm{NO}_{2}$ & $\mathbf{4 b}$ & 2 & 25 & 88 \\
3 & 3-NO2 & $\mathbf{4 c}$ & 2 & 25 & 78 \\
4 & 2,4-dichloro & $\mathbf{4 d}$ & 2 & 25 & 89 \\
5 & $4-\mathrm{Cl}$ & $\mathbf{4 e}$ & 2 & 25 & 84 \\
6 & $3-\mathrm{Cl}$ & $\mathbf{4 f}$ & 2 & 25 & 76 \\
7 & $2-\mathrm{Cl}$ & $\mathbf{4 g}$ & 2 & 25 & 92 \\
8 & $4-\mathrm{Me}$ & $\mathbf{4 h}$ & 2 & 25 & 77 \\
9 & $4-\mathrm{iPr}$ & $\mathbf{4 i}$ & 2 & 25 & 62 \\
10 & $4-\mathrm{OMe}$ & $\mathbf{4 j}$ & 2 & 25 & 79 \\
11 & $3-\mathrm{OMe}$ & $\mathbf{4 k}$ & 2 & 25 & 62 \\
12 & $2-\mathrm{KMe}$ & $\mathbf{4 l}$ & 2 & 70 & 76 \\
\hline
\end{tabular}

a Reaction conditions: The reaction was performed by using $2 \mathrm{mmol}$ of $\mathbf{1 a}-1,2 \mathrm{mmol}$ of $\mathbf{2}$, and $2 \mathrm{mmol}$ of 3 , in the presence of $5 \mathrm{~mol} \%$ of sodium carbonate in $10 \mathrm{~mL}$ of water at room temperature conditions.

b Isolated yields.

\section{Experimental}

Melting points were determined by open capillary method on STUART SMP3 instrument (BIBBY STERILIN, UK) and were uncorrected. IR spectra (KBr disc) were recorded on an Impact 410 FT-IR Spectrometer (Nicolet, USA). ${ }^{1} \mathrm{H}$ and ${ }^{13} \mathrm{C}$ NMR spectra were recorded on Avance Spectrometer AV500 (Bruker, Germany) at 500.13 and $125.77 \mathrm{MHz}$, respectively, using DMSO- $d_{6}$ as solvent and TMS as an internal standard. All analytical thin-layer chromatography (TLC) was performed on silica gel $60 \mathrm{WF}_{254} \mathrm{~S}$ aluminum sheets (Merck, Germany) and was visualized with UV light. Chemical reagents in high purity were purchased from the Merck Chemical Company. All materials were of commercial reagent grade.

\subsection{General Procedure for Synthesis of 7-Hydroxy-4H-Chromene-3-Carbonitriles and Analytical Data}

To a mixture of substituted benzaldehyde 1a-i $(2 \mathrm{mmol})$, malononitrile $2(2 \mathrm{mmol})$, and resorcinol $3(2 \mathrm{mmol})$, in $5 \mathrm{~mL}$ of water, was added a solution of sodium carbonate $(2.12 \mathrm{mg}, 0.5$ $\mathrm{mmol}$ ) in $5 \mathrm{~mL}$ of water. The reaction mixture was stirred for $2 \mathrm{~h}$ at room temperature (the reactions were monitored by TLC). Separated solid product was filtered, washed by water, and recrystallized from a mixture of $96 \%$ ethanol and toluene to afford the titled chromene-3-carbonitriles 4a-1. The characterizations of these 2-amino-7-hydroxy-4-aryl-4H-chromene-3-carbonitriles 4a-1 are as follows.

\subsubsection{2-Amino-7-Hydroxy-4-Phenyl-4H-Chromene-3-Carbonitrile (4a)}

Ivory white solids. M.p. $235-236^{\circ} \mathrm{C}$ (from $96 \%$ ethanol/toluene 1:1), ref [40]: $234-237^{\circ} \mathrm{C}$; IR (KBr), $v\left(\mathrm{~cm}^{-1}\right): 3500,3427,3350,2192,1647,1600,1520,1145,1104 ;{ }^{1} \mathrm{H}$ NMR $\left(500.13 \mathrm{MHz}, \mathrm{DMSO}-d_{6}\right), \delta$ (ppm): 9.70 (s, 1H, 7-OH), 7.31 (t, 2H, J= 7.5 Hz, H-3' \& H-5'), $7.21\left(\mathrm{t}, 1 \mathrm{H}, J=7.5 \mathrm{~Hz}, \mathrm{H}-4^{\prime}\right), 7.17(\mathrm{~d}, 2 \mathrm{H}$, $J=7.5 \mathrm{~Hz}, \mathrm{H}-2^{\prime}$ and $\left.\mathrm{H}-6^{\prime}\right), 6.86\left(\mathrm{~s}, 2 \mathrm{H}, 2-\mathrm{NH}_{2}\right), 6.81(\mathrm{~d}, 1 \mathrm{H}, J=8.5 \mathrm{~Hz}, \mathrm{H}-5), 6.49(\mathrm{dd}, 1 \mathrm{H}, J=8.5,2.5 \mathrm{~Hz}$, H-6), 6.42 (d, 1H, J = 2.5 Hz, H-8), 4.63 (s, 1H, H-4); ${ }^{13} \mathrm{C}$ NMR (125.77 MHz, DMSO-d 6 ), $\delta(\mathrm{ppm}): 160.7$ (C-2), 157.6 (C-7), 149.3 (C-8a), 146.8 (C-1'), 130.4 (C-5), 129.1 (C-3' and C-5'), 127.9 (C-2' and C-6'), 127.1 (C-4'), $121.1(\mathrm{C} \equiv \mathrm{N}), 114.2$ (C-4a), 112.9 (C-6), 102.7 (C-8), 56.8 (C-3), 40.5 (C-4).

\subsubsection{2-Amino-7-Hydroxy-4-(4-Nitrophenyl)-4H-Chromene-3-Carbonitrile (4b)}

Yellow crystals. M.p. $205-206^{\circ} \mathrm{C}$ (from $96 \%$ ethanol/toluene 1:1), ref [21]: 211-213 ${ }^{\circ} \mathrm{C}$; IR (KBr), $v\left(\mathrm{~cm}^{-1}\right): 3460,3335,3210,2188,1640,1582,1510,1346,1157,1103 ;{ }^{1} \mathrm{H}$ NMR $(500.13 \mathrm{MHz}$, DMSO-d6), $\delta(\mathrm{ppm}): 9.79(\mathrm{~s}, 1 \mathrm{H}, \mathrm{OH}), 8.20\left(\mathrm{~d}, 2 \mathrm{H}, J=8.75 \mathrm{~Hz}, \mathrm{H}-3^{\prime}\right.$ and $\left.\mathrm{H}-5^{\prime}\right), 7.46\left(\mathrm{~d}, 2 \mathrm{H}, J=8.75 \mathrm{~Hz}, \mathrm{H}-2^{\prime}\right.$ and $\mathrm{H}-$ $\left.6^{\prime}\right), 7.03\left(\mathrm{~s}, 2 \mathrm{H}, 2-\mathrm{NH}_{2}\right), 6.82(\mathrm{~d}, J=8.0 \mathrm{~Hz}, 1 \mathrm{H}, \mathrm{H}-5), 6.51$ (dd, J = 8.25, $\left.2.25 \mathrm{~Hz}, 1 \mathrm{H}, \mathrm{H}-6\right), 6.46(\mathrm{~d}, 1 \mathrm{H}, J$ $=2.5 \mathrm{~Hz}, \mathrm{H}-8), 4.87$ (s, 1H, H-4); ${ }^{13} \mathrm{C}$ NMR (125.77 MHz, DMSO-d6), $\delta$ (ppm): 160,9 (C-2); 158,0 (C-7); 
154,2 (C-8a); 149,4 (C-1'); 146,8 (C-4'); 130,4 (C-5); 129,2 (C-2' and C-6'); 124,5 (C-3' and C-5'); 120,8 $(\mathrm{C} \equiv \mathrm{N}) ; 113,1$ (C-4a); 112,8 (C-6); 102,9 (C-8); 55,6 (C-3); 40,1 (C-4).

\subsubsection{2-Amino-7-Hydroxy-4-(3-Nitrophenyl)-4H-Chromene-3-Carbonitrile (4c)}

Pale yellow crystals. M.p. $211-213{ }^{\circ} \mathrm{C}$ (from $96 \%$ ethanol/toluene 1:1), ref [21]: 189-191 ${ }^{\circ} \mathrm{C}$; IR $(\mathrm{KBr}), v\left(\mathrm{~cm}^{-1}\right): 3500,3429,3300,2188,1647,1582,1510,1346,1154,1047 ;{ }^{1} \mathrm{H}$ NMR $(500.13 \mathrm{MHz}$, DMSO- $\left.d_{6}\right), \delta(\mathrm{ppm}): 9.79(\mathrm{~s}, 1 \mathrm{H}, 7-\mathrm{OH}), 8.10\left(\mathrm{ddd}, J=8.0,2.0,1.5 \mathrm{~Hz}, 1 \mathrm{H}, \mathrm{H}-4^{\prime}\right), 8.04(\mathrm{t}, J=2.0 \mathrm{~Hz}, 1 \mathrm{H}$, H-2') $7.67\left(\mathrm{dt}, J=7.75,1.5 \mathrm{~Hz}, 1 \mathrm{H}, \mathrm{H}-6^{\prime}\right), 7.64\left(\mathrm{t}, J=7.75 \mathrm{~Hz}, 1 \mathrm{H}, \mathrm{H}-5^{\prime}\right), 7.04\left(\mathrm{~s}, 2 \mathrm{H}, 2-\mathrm{NH}_{2}\right), 6.86(\mathrm{~d}, J=$ $8.5 \mathrm{~Hz}, 1 \mathrm{H}, \mathrm{H}-5), 6.52$ (dd, J = 8.5, $2.5 \mathrm{~Hz}, 1 \mathrm{H}, \mathrm{H}-6), 6.46$ (d, J = $2.5 \mathrm{~Hz}, 1 \mathrm{H}, \mathrm{H}-8), 4.92(\mathrm{~s}, 1 \mathrm{H}, \mathrm{H}-4) ;{ }^{13} \mathrm{C}$ NMR (125.77 MHz, DMSO-d6), ठ (ppm): 161.0 (C-2), 158.0 (C-7), 149.4 (C-8a), 149.1 (C-3'), 148.4 (C-1'), 134.8 (C-6'), 130.8 (C-2'), 130.5 (C-5), $122.3\left(\mathrm{C}-5^{\prime}\right), 122.2\left(\mathrm{C}-4^{\prime}\right), 120.8(\mathrm{C} \equiv \mathrm{N}), 113.2(\mathrm{C}-4 \mathrm{a}), 113.0$ (C-6), 102.9 (C-8), 55.8 (C-3), 40.3 (C-4).

\subsubsection{2-Amino-7-Hydroxy-4-(2,4-Dichlorophenyl)-4H-Chromene-3-Aarbonitrile (4d)}

Pale yellow crystals. M.p. $189-191{ }^{\circ} \mathrm{C}$ (from $96 \%$ ethanol/toluene 1:1); ref [41]: 256-258 ${ }^{\circ} \mathrm{C}$; IR $(\mathrm{KBr}), v\left(\mathrm{~cm}^{-1}\right): 3500,3470,3300,3254,3050,2186,1628,1580,1503,1154,1046 ;{ }^{1} \mathrm{H}$ NMR $(500.13 \mathrm{MHz}$, DMSO-d 6 ), $\delta$ (ppm): 9.78 (s, 1H, 7-OH), 7.58 (d, J=2.0 Hz, 1H, H-3'), 7.40 (dd, J = 8.25, $2.0 \mathrm{~Hz}, 1 \mathrm{H}, \mathrm{H}-$ $\left.5^{\prime}\right), 7.22\left(\mathrm{~d}, J=8.25 \mathrm{~Hz}, 1 \mathrm{H}, \mathrm{H}-6^{\prime}\right), 6.97\left(\mathrm{~s}, 2 \mathrm{H}, 2-\mathrm{NH}_{2}\right), 6.73(\mathrm{~d}, J=8.5 \mathrm{~Hz}, 1 \mathrm{H}, \mathrm{H}-5), 6.50(\mathrm{dd}, J=8.5,2.5$ $\mathrm{Hz}, 1 \mathrm{H}, \mathrm{H}-6), 6.43$ (d, J = 2.5 Hz, 1H, H-8), 5.14 (s, 1H, H-4); ${ }^{13} \mathrm{C}$ NMR (125.77 MHz, DMSO-d 6 ), $\delta(\mathrm{ppm})$ : 161.0 (C-2), 158.0 (C-7), 149.5 (C-8a), 142.4 (C-1'), 133.3 (C-2'), 132.7 (C-4'), 132.6 (C-6'), 129.7 (C-3'), 129.6 (C-5), $128.5\left(\mathrm{C}-5^{\prime}\right), 120.6(\mathrm{C} \equiv \mathrm{N}), 113.1$ (C-4a), 112.4 (C-6), 102.8 (C-8), 54.9 (C-3), 37.4 (C-4).

\subsubsection{2-Amino-7-Hydroxy-4-(4-Chlorophenyl)-4H-Chromene-3-Carbonitrile (4e)}

Pale yellow crystals. M.p. $238-240{ }^{\circ} \mathrm{C}$ (from $96 \%$ ethanol/toluene 1:1), ref [40]: 162-164 ${ }^{\circ} \mathrm{C}$; IR $(\mathrm{KBr}), v\left(\mathrm{~cm}^{-1}\right): 3467,3341,3262,2191,1697,1640,1510,1157,1109 ;{ }^{1} \mathrm{H}$ NMR $(500.13 \mathrm{MHz}$, DMSO-d6), $\delta$ (ppm): $9.72(\mathrm{~s}, 1 \mathrm{H}, 7-\mathrm{OH}), 7.38\left(\mathrm{dt}, 2 \mathrm{H}, J=8.75,2.25 \mathrm{~Hz}, \mathrm{H}-2^{\prime}\right.$ and $\left.\mathrm{H}-6^{\prime}\right), 7.20(\mathrm{dt}, 2 \mathrm{H}, J=8.75,2.25$ $\mathrm{Hz}, \mathrm{H}-3^{\prime}$ and H-5'), 6.91 (s, 2H, 2-NH2), 6.79 (d, J= 8.5 Hz, 1H, H-5), 6.50 (dd, J = 8.5, 2.5 Hz, 1H, H-6), 6.41 (d, 1H, J = 2.5 Hz, H-8), 4.67 (s, 1H, H-4); ${ }^{13} \mathrm{C}$ NMR (125.77 MHz, DMSO- $\left.d_{6}\right), \delta(\mathrm{ppm}): 160.2$ (C-2), 157.2 (C-7), 148.8 (C-8a), 145.3 (C-1'), 131.2 (C-4'), 129.9 (C-5), 129.3 (C-2' and C-6'), 128.5 (C-3' and C5'), $120.5(\mathrm{C} \equiv \mathrm{N}), 113.2(\mathrm{C}-4 \mathrm{a}), 112.5(\mathrm{C}-6), 102.2(\mathrm{C}-8), 55.8$ (C-3), 39.0 (C-4).

\subsubsection{2-Amino-7-Hydroxy-4-(3-Chlorophenyl)-4H-Chromene-3-Carbonitrile (4f)}

Pale yellow crystals. M.p. $192-196{ }^{\circ} \mathrm{C}$ (from $96 \%$ ethanol/toluene 1:1); ref [41]: 106-109 ${ }^{\circ} \mathrm{C}$; IR $(\mathrm{KBr}), v\left(\mathrm{~cm}^{-1}\right): 3500,3465,3328,3254,3050,2190,1640,1580,1503,1154,1044 ;{ }^{1} \mathrm{H}$ NMR $(500.13 \mathrm{MHz}$, DMSO- $\left.d_{6}\right), \delta(\mathrm{ppm}): 9.81(\mathrm{~s}, 1 \mathrm{H}, 7-\mathrm{OH}), 7.35\left(\mathrm{t}, J=7.75 \mathrm{~Hz}, 1 \mathrm{H}, \mathrm{H}-5^{\prime}\right), 7.28(\mathrm{dt}, J=7.75,1.0 \mathrm{~Hz}, 1 \mathrm{H}, \mathrm{H}-$ 4'), 7.21 (br. s, 1H, H-2'), 7.15 (d, J = $\left.7.75 \mathrm{~Hz}, 1 \mathrm{H}, \mathrm{H}-6^{\prime}\right), 6.96\left(\mathrm{~s}, 2 \mathrm{H}, 2-\mathrm{NH}_{2}\right), 6.83(\mathrm{~d}, J=8.5 \mathrm{~Hz}, 1 \mathrm{H}, \mathrm{H}-$ 5), $6.51(\mathrm{dd}, J=8.5,2.0 \mathrm{~Hz}, 1 \mathrm{H}, \mathrm{H}-6), 6.43$ (d, J = $2.0 \mathrm{~Hz}, 1 \mathrm{H}, \mathrm{H}-8), 4.69$ (s, 1H, H-4); ${ }^{13} \mathrm{C}$ NMR (125.77 MHz. DMSO- $\left.d_{6}\right), \delta$ (ppm): 160.9 (C-2), 157.7 (C-7), 149.3 (C-8a), 149.3 (C-1'), 133.6 (C-3'), 131.1 (C-5'), 130.4 (C-5), 127.6 (C-2'), 127.2 (C-4'), 126.7 (C-6'), $121.0(\mathrm{C} \equiv \mathrm{N}), 113.5$ (C-4a), 113.0 (C-6), 102.8 (C-8), 56.1 (C-3), $49.1(\mathrm{C}-4)$.

\subsubsection{2-Amino-7-Hydroxy-4-(2-Chlorophenyl)-4H-Chromene-3-Carbonitrile (4g)}

Pale yellow crystals. M.p. $216-217{ }^{\circ} \mathrm{C}$ (from $96 \%$ ethanol/toluene 1:1); ref [21]: 184-186 ${ }^{\circ} \mathrm{C}$; IR (KBr), $v\left(\mathrm{~cm}^{-1}\right): 3465,3328,3254,3050,2190,1640,1580,1503,1154,1044 ;{ }^{1} \mathrm{H}$ NMR $(500.13 \mathrm{MHz}$, DMSO- $\left.d_{6}\right), \delta$ (ppm): 9.73 (s, 1H, 7-OH), $7.42\left(\mathrm{dd}, 1 \mathrm{H}, J=7.75,1.25 \mathrm{~Hz}, \mathrm{H}-3^{\prime}\right), 7.31$ (td, $1 \mathrm{H}, J=7.5,1.0$ $\left.\mathrm{Hz}, \mathrm{H}-6^{\prime}\right), 7.25$ (td, 1H, J = 7.75, $\left.1.75 \mathrm{~Hz}, \mathrm{H}-4^{\prime}\right), 7.19$ (dd, 1H, J = 7.5, $\left.2.0 \mathrm{~Hz}, \mathrm{H}-5^{\prime}\right), 6.92\left(\mathrm{~s}, 2 \mathrm{H}, 2-\mathrm{NH}_{2}\right)$, $6.74(\mathrm{~d}, 1 \mathrm{H}, J=8.5 \mathrm{~Hz}, \mathrm{H}-5), 6.48(\mathrm{dd}, 1 \mathrm{H}, J=8.5,2.5 \mathrm{~Hz}, \mathrm{H}-6), 6.41(\mathrm{~d}, 1 \mathrm{H}, J=2.5 \mathrm{~Hz}, \mathrm{H}-8), 5.14(\mathrm{~s}, 1 \mathrm{H}$, H-4); ${ }^{13} \mathrm{C}$ NMR (125.77 MHz, DMSO- $\left.d_{6}\right), \delta$ (ppm): 160.5 (C-2), 157.3 (C-7), 149.0 (C-8a), 142.8 (C-1'), $131.8(\mathrm{C}-5), 130.8\left(\mathrm{C}-2^{\prime}\right), 129.7\left(\mathrm{C}-3^{\prime}\right), 129.2\left(\mathrm{C}-4^{\prime}\right), 128.5\left(\mathrm{C}-5^{\prime}\right), 127.8\left(\mathrm{C}-6^{\prime}\right), 120.3(\mathrm{C} \equiv \mathrm{N}), 112.5(\mathrm{C}-6$ and C-4a), 102.2 (C-8), 54.9 (C-3), 37.2 (C-4). 


\subsubsection{2-Amino-7-Hydroxy-4-(4-Methylphenyl)-4H-Chromene-3-Carbonitrile (4h)}

Pale yellow solids. M.p. $208-210^{\circ} \mathrm{C}$ (from 96\% ethanol/toluene 2:1), ref [40]: 182-184 ${ }^{\circ} \mathrm{C}$; IR (KBr), $v\left(\mathrm{~cm}^{-1}\right): 3454,3320,3256,3200,2195,1638,1587,1503,1152,1047 ;{ }^{1} \mathrm{H}$ NMR $(500.13 \mathrm{MHz}$, DMSO-d6), $\delta$ (ppm): $9.72(\mathrm{~s}, 1 \mathrm{H}, 7-\mathrm{OH}), 7.17\left(\mathrm{~d}, 2 \mathrm{H}, J=8.0 \mathrm{~Hz}, \mathrm{H}-2^{\prime}\right.$ and $\left.\mathrm{H}-6^{\prime}\right), 7.11\left(\mathrm{~d}, 2 \mathrm{H}, J=8.0 \mathrm{~Hz}, \mathrm{H}-3^{\prime}\right.$ and H$\left.5^{\prime}\right), 6.88\left(\mathrm{~s}, 2 \mathrm{H}, 2-\mathrm{NH}_{2}\right), 6.84(\mathrm{~d}, 1 \mathrm{H}, J=8.5 \mathrm{~Hz}, \mathrm{H}-5), 6.49$ (dd, 1H, J = 8.5, 2.5 Hz, H-6), 6.46 (d, 1H, J = $2.5 \mathrm{~Hz}, \mathrm{H}-8), 4.63$ (s, 1H, H-4), 3.40 (s, 3H, 4'-CH3); ${ }^{13} \mathrm{C}$ NMR (125.77 MHz, DMSO-d 6 ), $\delta(p p m): 160.6$ (C-2), 157.5 (C-7), 149.3 (C-8a), 143.9 (C-1'), 136.2 (C-4'), 130.4 (C-5), 129.6 (C-2' and C-6'), 127.8 (C-3' and $\left.\mathrm{C}-5^{\prime}\right), 121.1(\mathrm{C} \equiv \mathrm{N}), 114.4(\mathrm{C}-4 \mathrm{a}), 112.8$ (C-6), 102.6 (C-8), 56.9 (C-3), 40.1 (C-4), $21.1\left(4^{\prime}-\mathrm{CH}_{3}\right)$.

\subsubsection{2-Amino-7-Hydroxy-4-(4-Isopropylphenyl)-4H-Chromene-3-Carbonitrile (4i)}

Ivory white crystals. M.p. $228-230{ }^{\circ} \mathrm{C}$ (from 96\% ethanol/toluene 2:1); IR (KBr), $v\left(\mathrm{~cm}^{-1}\right): 3313$,

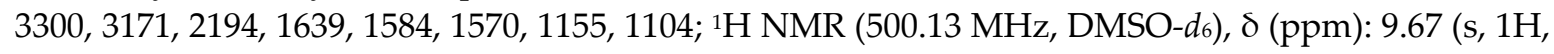
7-OH), $7.17\left(\mathrm{~d}, 2 \mathrm{H}, J=8.0 \mathrm{~Hz}, \mathrm{H}-2^{\prime}\right.$ and $\left.\mathrm{H}-6^{\prime}\right), 7.08\left(\mathrm{~d}, 2 \mathrm{H}, J=8.0 \mathrm{~Hz}, \mathrm{H}-3^{\prime}\right.$ and $\left.\mathrm{H}-5^{\prime}\right), 6.83(\mathrm{~s}, 2 \mathrm{H}, 2-$ $\left.\mathrm{NH}_{2}\right), 6.82(\mathrm{~d}, 1 \mathrm{H}, J=9.0 \mathrm{~Hz}, \mathrm{H}-5), 6.49(\mathrm{dd}, 1 \mathrm{H}, J=8.25,2.25 \mathrm{~Hz}, \mathrm{H}-6), 6.41(\mathrm{~d}, 1 \mathrm{H}, J=2.5 \mathrm{~Hz}, \mathrm{H}-8)$, 4.58 (s, 1H, H-4), 2.84 [septet, $1 \mathrm{H}, J=7.0 \mathrm{~Hz}, 3-\mathrm{CH}\left(\mathrm{CH}_{3}\right)_{2}, 1.18\left[\mathrm{~d}, 6 \mathrm{H}, J=7.0 \mathrm{~Hz}, 3-\mathrm{CH}\left(\mathrm{CH}_{3}\right)_{2}\right.$ ]; ${ }^{13} \mathrm{C}$ NMR (125.77 MHz, DMSO- $\left.d_{6}\right), \delta$ (ppm): 160.7 (C-2), 157.5 (C-7), 149.3 (C-8a), 147.1 (C-4'), 144.3 (C-1'), $130.4(\mathrm{C}-5), 127.7$ (C-2' and C-6'), $127.0\left(\mathrm{C}-3^{\prime}\right.$ and $\left.\mathrm{C}^{\prime} 5^{\prime}\right), 121.2(\mathrm{C} \equiv \mathrm{N}), 114.4(\mathrm{C}-4 \mathrm{a}), 112.8(\mathrm{C}-6), 102.6$ (C8), 56.9 (C-3), 39.5 (C-4), 33.5 [4'- $\left.\mathrm{CH}\left(\mathrm{CH}_{3}\right)_{2}\right], 24.3$ [4'- $\left.\mathrm{CH}\left(\mathrm{CH}_{3}\right)_{2}\right]$.

\subsubsection{2-Amino-7-Hydroxy-4-(4-Methoxyphenyl)-4H-Chromene-3-Carbonitrile (4j)}

Yellow crystals. M.p. $225-227^{\circ} \mathrm{C}$ (from 96\% ethanol/toluene 2:1); ref [40]: 110-112 ${ }^{\circ} \mathrm{C}$; IR (KBr), $v\left(\mathrm{~cm}^{-1}\right): 3550,3420,3358,2196,1642,1592,1570,1158,1110 ;{ }^{1} \mathrm{H}$ NMR $(500.13 \mathrm{MHz}$, DMSO-d 6$), \delta$ (ppm): $9.66(\mathrm{~s}, 1 \mathrm{H}, 7-\mathrm{OH}), 7.08\left(\mathrm{t}, 2 \mathrm{H}, J=8.25 \mathrm{~Hz}, \mathrm{H}-2^{\prime}\right.$ and $\left.\mathrm{H}-6^{\prime}\right), 6.87\left(\mathrm{t}, 2 \mathrm{H}, J=8.25 \mathrm{~Hz}, \mathrm{H}-3^{\prime}\right.$ and $\mathrm{H}-$ $\left.5^{\prime}\right), 6.80\left(\mathrm{~s}, 2 \mathrm{H}, 2-\mathrm{NH}_{2}\right), 6.78$ (d, J = 8.5 Hz, 1H, H-5), 6.48 (dd, J = 8.5, $\left.2.5 \mathrm{~Hz}, 1 \mathrm{H}, \mathrm{H}-6\right), 6.40(\mathrm{~d}, 1 \mathrm{H}, J=$ $2.5 \mathrm{~Hz}, \mathrm{H}-8), 4.57$ (s, 1H, H-4), 3.72 (s, 3H, 4-OCH 3 ); ${ }^{13} \mathrm{C}$ NMR (125.77 MHz, DMSO-d6), $\delta$ (ppm): 160.1 (C-2), 158.0 (C-7), 156.9 (C-8a), 148.8 (C-4'), 138.5 (C-1'), 129.9 (C-5), $128.4\left(\mathrm{C}-2^{\prime}\right.$ and C-6'), $120.7(\mathrm{C} \equiv \mathrm{N})$, 114.1 (C-4a), 113.9 (C-3' and C-5'), 112.3 (C-6), 102.1 (C-8), 56.6 (C-3), $55.0\left(4^{\prime}-\mathrm{OCH}_{3}\right), 39.0(\mathrm{C}-4)$.

\subsubsection{2-Amino-7-Hydroxy-4-(3-Methoxyphenyl)-4H-Chromene-3-Carbonitrile (4k)}

Yellow crystals. M.p. $177-179{ }^{\circ} \mathrm{C}$ (from 96\% ethanol/toluene 1:1); IR (KBr), $v\left(\mathrm{~cm}^{-1}\right)$ : 3550, 3420, 3358, 2196, 1642, 1592, 1571, 1151, 1035; ${ }^{1} \mathrm{H}$ NMR (500.13 MHz, DMSO-d6), $\delta$ (ppm): 9.68 (s, 1H, 7-OH), $7.22\left(\mathrm{t}, 1 \mathrm{H}, J=7.25 \mathrm{~Hz}, \mathrm{H}-5^{\prime}\right), 6.84\left(\mathrm{~s}, 2 \mathrm{H}, 2-\mathrm{NH}_{2}\right), 6.83\left(\mathrm{~d}, 1 \mathrm{H}, J=7.5 \mathrm{~Hz}, \mathrm{H}-4^{\prime}\right), 6.78(\mathrm{dd}, 1 \mathrm{H}, J=7.0,2.0$ Hz, H-6'), 6.73 (s, 1H, H-2'), 6.72 (d, 1H, J = 7.5 Hz, H-5), 6.49 (dd, 1H, J = 8.25, 2.25 Hz, H-6), 6.41 (d, $1 \mathrm{H}, J=2.0 \mathrm{~Hz}, \mathrm{H}-8), 4.59$ (s, 1H, H-4), 3.34 (s, 3H, 3'-OCH $) ;{ }^{13} \mathrm{C} \mathrm{NMR}\left(125.77 \mathrm{MHz}, \mathrm{DMSO}-d_{6}\right), \delta(\mathrm{ppm}):$ 160.3 (C-2), 159.3 (C-7), 157.1 (C-3'), 148.8 (C-8a), 147.9 (C-1'), 129.8 (C-5), 129.7 (C-5'), 120.6 (C-6'), $119.6(\mathrm{C} \equiv \mathrm{N}), 113.6$ (C-4a), 113.5 (C-2'), 112.3 (C-4'), 111.5 (C-6), 102.1 (C-8), 54.9 (C-3), 56.1 (C-3), 54.9 $\left(3^{\prime}-\mathrm{OCH}_{3}\right), 39.9(\mathrm{C}-4)$.

\subsubsection{2-Amino-7-Hydroxy-4-(2-Methoxyphenyl)-4H-Chromene-3-Carbonitrile (41)}

Ivory white crystals. M.p. $218-220{ }^{\circ} \mathrm{C}$ (from $96 \%$ ethanol/toluene 2:1), ref [21]: 222-224 ${ }^{\circ} \mathrm{C}$; IR $(\mathrm{KBr}), v\left(\mathrm{~cm}^{-1}\right): 3477,3340,3250,3191,2203,1643,1587,1502,1157,1053 ;{ }^{1} \mathrm{H}$ NMR $(500.13 \mathrm{MHz}$, DMSO- $\left.d_{6}\right), \delta(\mathrm{ppm}): 9.62(\mathrm{~s}, 1 \mathrm{H}, 7-\mathrm{OH}), 7.19\left(\mathrm{td}, 1 \mathrm{H}, J=8.0,1.5 \mathrm{~Hz}, \mathrm{H}-6^{\prime}\right), 6.99(\mathrm{td}, 1 \mathrm{H}, J=7.5,1.5 \mathrm{~Hz}$, $\left.\mathrm{H}-5^{\prime}\right), 6.89\left(\mathrm{td}, 1 \mathrm{H}, J=7.25,1.0 \mathrm{~Hz}, \mathrm{H}-4^{\prime}\right), 6.84(\mathrm{~d}, 1 \mathrm{H}, J=9.0 \mathrm{~Hz}, \mathrm{H}-5), 6.78\left(\mathrm{~s}, 2 \mathrm{H}, 2-\mathrm{NH}_{2}\right), 6.74(\mathrm{~d}, 1 \mathrm{H}$, $J=8.5 \mathrm{~Hz}, \mathrm{H}-5), 6.46$ (dd, 1H, J = 8.5, $2.5 \mathrm{~Hz}, \mathrm{H}-6), 6.39$ (d, 1H, J = 2.5 Hz, H-8), 4.99 (s, 1H, H-4), 3.34 (s, 3H, 2'-OCH 3 ); ${ }^{13} \mathrm{C}$ NMR (125.77 MHz, DMSO- $\left.d_{6}\right), \delta(\mathrm{ppm}): 161.4$ (C-2), 157.4 (C-7), 156.8 (C-2'), 149.6 (C-8a), 134.6 (C-5), 129.7 (C-6'), $129.0\left(\mathrm{C}-4^{\prime}\right), 128.4\left(\mathrm{C}-5^{\prime}\right), 121.2(\mathrm{C} \equiv \mathrm{N}), 114.5\left(\mathrm{C}-1^{\prime}\right), 112.7$ (C-4a), 112.0 (C-6), 102.6 (C-8), $56.1(\mathrm{C}-3), 55.8\left(2^{\prime}-\mathrm{OCH}_{3}\right), 34.0$ (C-4). 


\section{Conclusions}

The use of sodium carbonate, as a catalyst in water, as the reaction medium in the synthesis of 2-amino-7-hydroxy-4H-chromene-3-carbonitriles 4a-1 opens a new direction in chromene chemistry. This synthetic procedure for these chromene-3-carbonitrile rings is a cheap, efficient, and simple method.

Acknowledgments. This research is funded by the Vietnam National Foundation for Science and Technology Development (NAFOSTED) under grant number 104.01-2017.02.

\section{References}

1. Bugaut, X.; Constantieux, T.; Coquerel, Y.; Rodriguez, J. 1,3-Dicarbonyls in multicomponent reactions. In Multicomponent Reactions in Organic Synthesis; Zhu, J., Wang, Q., Wang, M.-X., Eds.; Wiley-VCH Verlag GmbH \& Co. KGaA: Weinheim, Germany, 2014; pp. 109-158.

2. Strecker, A. Ueber die künstliche Bildung der Milchsäure und einen neuen, dem Glycocoll homologen Körper. Justus Liebigs Annalen der Chemie 1850, 75, 27-45, doi:10.1002/jlac.18500750103.

3. Cioc, R.C.; Ruijter, E.; Orru, R.V.A. Multicomponent reactions: Advanced tools for sustainable organic synthesis. Green Chem. 2014, 16, 2958-2975, doi:10.1039/C4GC00013G.

4. Ibarra, I.A.; Islas-Jacome, A.; Gonzalez-Zamora, E. Synthesis of polyheterocycles via multicomponent reactions. Org. Biomol. Chem. 2018, 16, 1402-1418, doi:10.1039/C7OB02305G.

5. Azizian, J.; Delbari, A.S.; Yadollahzadeh, K. One-Pot, Three-Component Synthesis of Pyrimido[4 ,5b]quinoline-tetraone Derivatives in Water. Synth. Commun. 2014, 44, 3277-3286, doi:10.1080/00397911.2011.626139.

6. Butler, R.N.; Coyne, A.G. Organic synthesis reactions on-water at the organic-liquid water interface. Org. Biomol. Chem. 2016, 14, 9945-9960, doi:10.1039/C6OB01724J.

7. Brahmachari, G. Green synthetic approaches for biologically relevant 2-amino-4H-pyrans and 2-amino-4Hpyran-annulated heterocycles in aqueous media. In Green Synthetic Approaches for Biologically Relevant Heterocycles; Brahmachari, G., Ed.; Elsevier Inc.: Amsterdam, The Netherlands; Oxford, UK; Waltham, MS, USA, 2015; pp. 185-208.

8. Chanda, A.; Fokin, V.V. Organic Synthesis "On Water". Chem. Rev. 2009, 109, 725-748, doi:10.1021/cr800448q.

9. Brahmachari, G. Room Temperature Organic Synthesis; Elsevier Inc.: Amsterdam, The Netherlands; Oxford, UK; Waltham, MS, USA, 2015; pp. 1-240.

10. Patil, S.A.; Patil, R.; Pfeffer, L.M.; Miller, D.D. Chromenes: Potential new chemotherapeutic agents for cancer. Future Med. Chem. 2013, 5, 1647-1660, doi:10.4155/fmc.13.126.

11. Subbareddy, C.V.; Sundarrajan, S.; Mohanapriya, A.; Subashini, R.; Shanmugam, S. Synthesis, antioxidant, antibacterial, solvatochromism and molecular docking studies of indolyl-4H-chromene-phenylprop-2-en1-one derivatives. J. Mol. Liq. 2018, 251, 296-307, doi:10.1016/j.molliq.2017.12.082.

12. Sashidhara, K.V.; Kumar, M.; Modukuri, R.K.; Srivastava, A.; Puri, A. Discovery and synthesis of novel substituted benzocoumarins as orally active lipid modulating agents. Biorg. Med. Chem. Lett. 2011, 21, 6709-6713, doi:10.1016/j.bmcl.2011.09.053.

13. Chung, S.-T.; Huang, W.-H.; Huang, C.-K.; Liu, F.-C.; Huang, R.-Y.; Wu, C.-C.; Lee, A.-R. Synthesis and anti-inflammatory activities of $4 \mathrm{H}$-chromene and chromeno[2,3-b]pyridine derivatives. Res. Chem. Intermed. 2016, 42, 1195-1215, doi:10.1007/s11164-015-2081-7.

14. Rajanarendar, E.; Reddy, M.N.; Krishna, S.R.; Murthy, K.R.; Reddy, Y.N.; Rajam, M.V. Design, synthesis, antimicrobial, anti-inflammatory and analgesic activity of novel isoxazolyl pyrimido[4,5-b]quinolines and isoxazolyl chromeno[2,3-d]pyrimidin-4-ones. Eur. J. Med. Chem. 2012, 55, 273-283, doi:10.1016/j.ejmech.2012.07.029.

15. El-Agrody, A.M.; Halawa, A.H.; Fouda, A.M.; Al-Dies, A.-A.M. The anti-proliferative activity of novel 4Hbenzo[ $h]$ chromenes, $7 H$-benzo[ $h]$-chromeno[2,3- $d]$ pyrimidines and the structure-activity relationships of the 2-, 3-positions and fused rings at the 2, 3-positions. J. Saudi Chem. Soc. 2017, 21, 82-90, doi:10.1016/j.jscs.2016.03.002.

16. Nancy, T.; Mary, Z.S.; Prasanna, R. 4-Aryl-4H-Chromene-3-Carbonitrile Derivates: Synthesis and Preliminary Anti-Breast Cancer Studies. J. Heterocycl. Chem. 2016, 53, 1778-1782, doi:10.1002/jhet.2483. 
17. Zghab, I.; Trimeche, B.; Mansour, M.B.; Hassine, M.; Touboul, D.; Jannet, H.B. Regiospecific synthesis, antibacterial and anticoagulant activities of novel isoxazoline chromene derivatives. Arab. J. Chem. 2017, 10, S2651-S2658, doi:10.1016/j.arabjc.2013.10.008.

18. Foroumadi, A.; Emami, S.; Sorkhi, M.; Nakhjiri, M.; Nazarian, Z.; Heydari, S.; Ardestani, S.K.; Poorrajab, F.; Shafiee, A. Chromene-Based Synthetic Chalcones as Potent Antileishmanial Agents: Synthesis and Biological Activity. Chem. Biol. Drug Des. 2010, 75, 590-596, doi:10.1111/j.1747-0285.2010.00959.x.

19. Soni, R.; Durgapal, S.D.; Soman, S.S.; Georrge, J.J. Design, synthesis and anti-diabetic activity of chromen2-one derivatives. Arab. J. Chem. 2016, doi:10.1016/j.arabjc.2016.11.011.

20. Reddy, B.V.S.; Divya, B.; Swain, M.; Rao, T.P.; Yadav, J.S.; Vardhan, M.V.P.S.V. A domino Knoevenagel hetero-Diels-Alder reaction for the synthesis of polycyclic chromene derivatives and evaluation of their cytotoxicity. Biorg. Med. Chem. Lett. 2012, 22, 1995-1999, doi:10.1016/j.bmcl.2012.01.033.

21. Javanshir, S.; Safari, M.; Dekamin, M.G. A facile and green three-component synthesis of 2-amino-3-cyano7-hydroxy-4H-chromenes on grinding. Scientia Iranica 2014, 21, 742-747.

22. Keerthy, H.K.; Garg, M.; Mohan, C.D.; Madan, V.; Kanojia, D.; Shobith, R.; Nanjundaswamy, S.; Mason, D.J.; Bender, A.; Rangappa, K.S.; et al. Synthesis and Characterization of Novel 2-Amino-ChromeneNitriles that Target Bcl-2 in Acute Myeloid Leukemia Cell Lines. PLoS ONE 2014, 9, e107118, doi:10.1371/journal.pone.0107118.

23. Pourmohammad, M.; Mokhtary, M. $\mathrm{K}_{2} \mathrm{CO}_{3}$-catalyzed synthesis of 2-amino-3-cyano- $4 H$-chromene derivatives with different substituents in water. C. R. Chim. 2015, 18, 554-557, doi:10.1016/j.crci.2014.09.008.

24. Kolla, S.R.; Lee, Y.R. Ca(OH)2-mediated efficient synthesis of 2-amino-5-hydroxy-4H-chromene derivatives with various substituents. Tetrahedron 2011, 67, 8271-8275, doi:10.1016/j.tet.2011.08.086.

25. Zavar, S. A novel three component synthesis of 2-amino-4H-chromenes derivatives using nano $\mathrm{ZnO}$ catalyst. Arab. J. Chem. 2017, 10, S67-S70, doi:10.1016/j.arabjc.2012.07.011.

26. Safari, J.; Zarnegar, Z.; Heydarian, M. Practical, ecofriendly, and highly efficient synthesis of 2-amino-4Hchromenes using nanocrystalline $\mathrm{MgO}$ as a reusable heterogeneous catalyst in aqueous media. J. Taibah Univ. Sci. 2013, 7, 17-25, doi:10.1016/j.jtusci.2013.03.001.

27. Magar, R.L.; Thorat, P.B.; Jadhav, V.B.; Tekale, S.U.; Dake, S.A.; Patil, B.R.; Pawar, R.P. Silica gel supported polyamine: A versatile catalyst for one pot synthesis of 2-amino-4H-chromene derivatives. J. Mol. Catal. A Chem. 2013, 374-375, 118-124, doi:10.1016/j.molcata.2013.03.022.

28. Kanakaraju, S.; Prasanna, B.; Basavoju, S.; Chandramouli, G.V.P. Ammonium acetate catalyzed an efficient one-pot three component synthesis of pyrano[3,2-c]chromene derivatives. Arab. J. Chem. 2017, 10, S2705S2713, doi:10.1016/j.arabjc.2013.10.014.

29. Kiyani, H.; Ghorbani, F. Potassium phthalimide promoted green multicomponent tandem synthesis of 2amino-4H-chromenes and 6-amino-4H-pyran-3-carboxylates. J. Saudi Chem. Soc. 2014, 18, 689-701, doi:10.1016/j.jscs.2014.02.004.

30. Ghorbani, M.; Noura, S.; Oftadeh, M.; Zolfigol, M.A.; Soleimani, M.H.; Behbodi, K. Preparation of neutral ionic liquid [2-Eim] OAc with dual catalytic-solvent system roles for the synthesis of 2-amino-3-cyano-7hydroxy-4-(aryl)-4H-chromene derivatives. J. Mol. Liq. 2015, 212, 291-300, doi:10.1016/j.molliq.2015.09.024.

31. Zheng, J.; Li, Y. Basic ionic liquid-catalyzed multicomponent synthesis of tetrahydrobenzo[b]pyrans and pyrano[c]chromenes. Mendeleev Commun. 2011, 21, 280-281, doi:10.1016/j.mencom.2011.09.017.

32. Akocak, S.; Şen, B.; Lolak, N.; Şavk, A.; Koca, M.; Kuzu, S.; Şen, F. One-pot three-component synthesis of 2-Amino-4H-Chromene derivatives by using monodisperse Pd nanomaterials anchored graphene oxide as highly efficient and recyclable catalyst. Nano-Struct. Nano-Objects 2017, 11, 25-31, doi:10.1016/j.nanoso.2017.06.002.

33. Şen, B.; Lolak, N.; Paralı, Ö.; Koca, M.; Şavk, A.; Akocak, S.; Şen, F. Bimetallic PdRu/graphene oxide based Catalysts for one-pot three-component synthesis of 2-amino-4H-chromene derivatives. Nano-Struct. NanoObjects 2017, 12, 33-40, doi:10.1016/j.nanoso.2017.08.013.

34. Patel, D.S.; Avalani, J.R.; Raval, D.K. One-pot solvent-free rapid and green synthesis of 3,4dihydropyrano[c]chromenes using grindstone chemistry. J. Saudi Chem. Soc. 2016, 20, S401-S405, doi:10.1016/j.jscs.2012.12.008.

35. Li, L.-Y.; Zeng, Q.-Q.; Yang, Y.-X.; Hu, H.-F.; Xu, M.; Guan, Z.; He, Y.-H. A domino reaction for the synthesis of 2-amino-4H-chromene derivatives using bovine serum albumin as a catalyst. J. Mol. Catal. B Enzym. 2015, 122, 1-7, doi:10.1016/j.molcatb.2015.08.005. 
36. Khaksar, S.; Rouhollahpour, A.; Talesh, S.M. A facile and efficient synthesis of 2-amino-3-cyano-4Hchromenes and tetrahydrobenzo[b]pyrans using 2,2,2-trifluoroethanol as a metal-free and reusable medium. J. Fluor. Chem. 2012, 141, 11-15, doi:10.1016/j.jfluchem.2012.05.014.

37. Safari, J.; Heydarian, M.; Zarnegar, Z. Synthesis of 2-amino-7-hydroxy-4H-chromene derivatives under ultrasound irradiation: A rapid procedure without catalyst. Arab. J. Chem. 2017, 10, S2994-S3000, doi:10.1016/j.arabjc.2013.11.038.

38. Safari, J.; Javadian, L. Ultrasound assisted the green synthesis of 2-amino-4H-chromene derivatives catalyzed by $\mathrm{Fe}_{3} \mathrm{O}_{4}$-functionalized nanoparticles with chitosan as a novel and reusable magnetic catalyst. Ultrason. Sonochem. 2015, 22, 341-348, doi:10.1016/j.ultsonch.2014.02.002.

39. Datta, B.; Pasha, M.A. Glycine catalyzed convenient synthesis of 2-amino-4H-chromenes in aqueous medium under sonic condition. Ultrason. Sonochem. 2012, 19, 725-728, doi:10.1016/j.ultsonch.2012.01.006.

40. Makarem, S.; Mohammadi, A.A.; Fakhari, A.R. A multi-component electro-organic synthesis of 2-amino4H-chromenes. Tetrahedron Lett. 2008, 49, 7194-7196, doi:10.1016/j.tetlet.2008.10.006.

41. Safari, J.; Zarnegar, Z.; Heydarian, M. Magnetic $\mathrm{Fe}_{3} \mathrm{O}_{4}$ Nanoparticles as Efficient and Reusable Catalyst for the Green Synthesis of 2-Amino-4H-chromene in Aqueous Media. Bull. Chem. Soc. Jpn. 2012, 85, 1332-1338, doi:10.1246/bcsj.20120209.

(C) 2018 by the authors. Licensee MDPI, Basel, Switzerland. This article is an open access article distributed under the terms and conditions of the Creative Commons Attribution (CC BY) license (http://creativecommons.org/licenses/by/4.0/). 